\title{
Surgical treatments of cystic neoplasms of the pancreas: an Asian university hospital experience
}

\author{
Suvit Sriussadaporn, Sukanya Sriussadaporn, Rattaplee Pak-art, Kritaya Kritayakirana, Supparerk Prichayudh, \\ Pasurachate Samorn \\ Department of Surgery, Faculty of Medicine, Chulalongkorn University, Bangkok 10330, Thailand
}

\begin{abstract}
Background: Premalignant and malignant cystic neoplasms of the pancreas are relatively rare, but absolute indications for surgical resection. Modern imaging modalities have increased recognition of asymptomatic cysts resulting in therapeutic dilemmas of whether surgery or observation is appropriate.

Objectives: To examine our surgical experience with cystic neoplasms of the pancreas.

Methods: A retrospective study of patients who had cystic neoplasms of the pancreas and underwent surgical resections from June 2000 to April 2013. Presenting symptoms, surgical procedures, pathological diagnoses, and postoperative complications were analyzed.

Results: Data from 16 patients were examined. Two patients had asymptomatic cystic neoplasms. Fourteen had symptoms ranging from 2 days to 6 years before diagnosis and surgery. Six patients underwent pylorus preserving pancreaticoduodenectomy, 4 underwent distal pancreatectomy with splenectomy, 2 underwent splenic preserving distal pancreatectomy, and 1 each underwent a classical Whipple operation, total pancreatectomy, distal pancreatectomy with splenectomy with partial resection of the posterior gastric wall, and distal pancreatectomy with splenectomy with left colectomy. The operative time ranged from 150 to 450 minutes. Operative blood transfusion ranged from 0 to 5 units. Four patients had mucinous cystadenoma, 4 had intraductal papillary mucinous neoplasia with varying degree of dysplasia and carcinomatous changes, 6 had other malignancies, and 2 had other benign cysts. Postoperative complications occurred in 3 patients. There was no perioperative mortality.

Conclusion: Any suspicion of malignant changes in asymptomatic cysts should have them considered for surgical resection. Meticulous surgical techniques are important for pancreatic resection to minimize the occurrence of postoperative complications.
\end{abstract}

Keywords: cystic neoplasms of the pancreas, intraductal papillary mucinous neoplasia, mucinous cystadenoma, pancreatic adenocarcinoma, pancreatic resection

Cystic neoplasms of the pancreas have been reported relatively uncommonly before the current advent of modern imaging modalities such as endoscopic ultrasonography (EUS), computed tomography (CT), and magnetic resonance imaging (MRI); with an incidence of $1 \%-5 \%$ of all primary pancreatic tumors $[1,2]$. According to the WHO classification of pancreatic cystic neoplasms, these lesions have been grouped into serous cystic neoplasms, mucinous cystic neoplasms, intraductal papillary mucinous neoplasms (IPMNs), and solid pseudopapillary neoplasms [3]. Most serous cystic neoplasms are benign and malignant serous cystadenocarcinomas are extremely rare $[4,5]$.

Correspondence to: Suvit Sriussadaporn, Department of Surgery, Faculty of Medicine, Chulalongkorn University, Bangkok 10330, Thailand.E-mail: Suvit.s@chula.ac.th
Mucinous cystic neoplasms are now the most common pancreatic cysts [6] and, with very few exceptions, are considered premalignant or malignant [7-9]. IPMNs are subcategorized clinically into main-duct, branch-duct, and mixed type IPMNs. The histology of these mucin-secreting neoplastic proliferations of the pancreatic duct varied from benign adenoma to borderline and malignant (in situ carcinoma and invasive carcinoma). Solid pseudopapillary neoplasm is a rare tumor with low malignant potential. It has been reported under various names including solid and cystic tumor, and solid and papillary epithelial neoplasm. Principle of management of these cystic neoplasms are straight forward; i.e. symptomatic, premalignant, and malignant cystic neoplasms should be surgically removed or resected. Identifying the premalignant lesion with subsequent resection is the most gratifying objective when dealing with these 
cystic neoplasms; because the prognosis is far superior compared with prognosis after resection of the adenocarcinoma of the pancreas. Furthermore, the 5-year survival after curative resection of the mucinous cystadenocarcinoma is also much better than after curative resection of the adenocarcinoma of the pancreas [2, 10-12]. However, surgery on the pancreas, e.g. pancreaticoduodenectomy, distal pancreatectomy with or without splenectomy, or even less aggressive procedures such as enucleation, are well-known for their association with morbidity and rarely mortality [13]. Therefore, accurate preoperative diagnosis and careful decision making with surgical indications are of utmost importance in management of patients with cystic neoplasms of the pancreas.

Recently, it has been universally accepted that the incidence of cystic neoplasms of the pancreas has increased much more than previously appreciated because of the increasing use of EUS, CT, and MRI $[6,14-16]$. This results in more asymptomatic cysts of the pancreas being diagnosed. The incidental finding of these cysts leads to a requirement for appropriate evaluation to search for the premalignant or malignant lesions that will benefit from resections. For asymptomatic benign lesions, they may be left alone and followed up to avoid complications from unnecessary operations. The purpose of this study is to examine patients who have cystic neoplasm of the pancreas and underwent surgical resection at our institution. Details of the study included demographic data, symptoms, preoperative diagnosis, indications for surgery, type of operation, postoperative complications, final pathological diagnoses, hospital stays, follow-up period, and status of patients at most recent follow up.

\section{Methods}

A retrospective study was performed of patients who had cystic neoplasms of the pancreas and underwent surgical removal by the first author at King Chulalongkorn Memorial Hospital, Bangkok, Thailand. The study period was from June 2000 to April 2013. Approval of the study was granted by the Institutional Review Board of the Faculty of Medicine, Chulalongkorn University, Bangkok, Thailand. The research committee (IRB) provided an exemption for written patient's approval for publication of their data in this retrospective study. All patients had provided informed consent for their surgery on admission.
During the study period, diagnosis of cystic neoplasms of the pancreas were made from CT and/ or MR imaging. In asymptomatic patients, diagnoses were made after routine medical check-up with abnormal findings on the pancreas on abdominal ultrasonography and subsequently confirmed by CT and/or MR imaging. Indications for surgical removal of the cystic neoplasms of the pancreas were 1 . symptomatic cysts, and 2. suspicion of malignancy or premalignancy. The operations were conducted on an elective basis. Lesions at the pancreatic head underwent pancreaticoduodenectomy with or without preservation of the pylorus. Lesions at the pancreatic body and tail were removed by distal pancreatectomy with or without splenectomy. No patient underwent preoperative or intraoperative needle aspiration cytology, needle biopsy, or incisional biopsy. During the operations; after the surgical specimens were removed, a frozen section of the resected margin was performed when negative margin for malignancy was doubtful. When a frozen section revealed a cancer positive result, further resection of the pancreas was performed. In one patient, a positive result for malignancy from a frozen section of the transected pancreatic body after pancreaticoduodenectomy led to completion of the operation by total pancreatectomy. The final pathological reports for this patient revealed IPMN of the entire main pancreatic duct.

Postoperatively, pathological reports of all patients were examined and analyzed. Postoperative complications were searched and treated individually. On or after postoperative day 3, the drainage fluid from the abdominal drain was routinely sent for measurement of the amylase level simultaneously with measurement of the serum amylase activities. Diagnosis of pancreatic fistula was made when fluid from the abdominal drain contained amylase more than 3 times the serum amylase level [17]. Postoperative mortality was defined as death within one month of the operation. After the patients were discharged, follow-up was regularly done until April 2013 or until the patients died. Interval CT was performed every 3-6 months in patients who had malignant cystic neoplasms to detect recurrence. CT was performed at variable periods depending on risk of recurrence in patients who had premalignant cystic neoplasms. The management of such recurrent malignant cystic neoplasms was also described. 


\section{Results}

During a 13-year-period, 16 patients entered into the study. Thirteen were women, and 3 were men. Their age ranged from 34 to 92 years (median 59.5, mean $57.3 \pm 17.6$ ). Two patients were asymptomatic and diagnoses were made from routine medical checkup and subsequent imaging study. Fourteen had symptoms leading to diagnostic evaluation and imaging study. Five patients presented with symptom of abdominal discomfort due to pressure effect of large abdominal mass. Four presented with abdominal pain. The remaining 5 symptomatic patients presented with the findings shown in Table 1. Duration of symptoms ranged from 2 days to 6 years before cystic neoplasms of the pancreas were diagnosed and resected. All patients underwent pancreatic resection on elective basis. The operative procedures were as shown in Table 2. The operative time ranged from 150 to 450 minutes (median 300, mean $293 \pm 98$ ). The operative blood transfusion ranged from 0 to 5 units (median 1.5 , mean $1.56 \pm 1.59$ ).

The size of the cystic neoplasms measuring at the greatest dimension from the surgical specimens after pancreatic resections ranged from 2.2 to 17.5 cm (median 10, mean $9.2 \pm 4.6$ ). Two patients had small neoplastic cysts, 2.2 and $2.5 \mathrm{~cm}$; one was asymptomatic, and one presented with epigastric pain for 6 years. The final pathological reports of both patients were IPMN of the pancreatic duct; one with severe dysplasia, one with carcinoma change. Definite diagnosis of the resected cystic neoplasms were as presented in Table 3.

Table 1. Symptoms of patients presented with cystic neoplasms of the pancreas

\begin{tabular}{lc}
\hline Symptom & Number of patients \\
\hline Asymptomatic & 2 \\
Abdominal discomfort (due to mass effect) & 5 \\
Abdominal pain & 4 \\
Fever, abdominal pain, and diabetes mellitus & 1 \\
Fever and abdominal pain & 1 \\
Abdominal discomfort and abdominal pain & 1 \\
Jaundice and weight loss & 1 \\
Cholangitis & 1 \\
Total & 16 \\
\hline
\end{tabular}

Table 2. Operative procedures for resection of cystic neoplasms of the pancreas

\begin{tabular}{lc}
\hline Operative procedure & Number of patients \\
\hline Pylorus preserving pancreaticoduodenectomy & 6 \\
Distal pancreatectomy with splenectomy & 4 \\
Splenic preserving distal pancreatectomy & 2 \\
Classical Whipple operation & 1 \\
Total pancreatectomy & 1 \\
Distal pancreatectomy with splenectomy with & 1 \\
$\quad$ partial resection of posterior gastric wall & \\
Distal pancreatectomy with splenectomy with & 1 \\
$\quad$ left colectomy & $16(100)$ \\
Total & \\
\hline
\end{tabular}


Table 3. Definite pathological diagnoses of cystic neoplasms of the pancreas

\begin{tabular}{lc}
\hline Definite pathological diagnosis & Number of patients \\
\hline Mucinous cystadenoma & 4 \\
Well differentiated neuroendocrine tumor & 2 \\
Adenocarcinoma of the pancreas & 2 \\
Mucinous cystadenocarcinoma & 1 \\
Solid pseudopapillary tumor & 1 \\
Hemorrhagic cyst & 1 \\
Benign epithelial cyst & 1 \\
Intraductal tubular neoplasm with low grade & \\
$\quad$ mild to severe dysplasias (PanIN grade 1-3) & 1 \\
IPMN* (moderately dysplasia with local severe dysplasia) & 1 \\
IMPM* (with focal carcinoma change) & 1 \\
IMPN* (with invasive well differentiated adenocarcinoma) & 1 \\
Total & 16 \\
\hline
\end{tabular}

IPMN* = Intraductal papillary mucinous neoplasia

Postoperative complications occurred in 3 patients; one had congestive heart failure, one had grade A pancreatic fistula, and one, who underwent total pancreatectomy, had diabetes mellitus. All had uneventful recovery after conservative treatment and appropriate medications. There was no postoperative mortality. The hospital stays ranged from 7 to 54 days (median 13, mean 17.9 \pm 12.1 ). The follow-up time ranged from 1 to 155 months (median 69, mean $63.8 \pm 54.8$ ). Two patients died at 3.5 and 11 months postoperatively; one had poorly differentiated adenocarcinoma of the pancreas, the other had cystadenocarcinoma of the pancreas. Thirteen patients were alive and well without evidence of pancreatic malignancy at the time of preparing this manuscript. One patient who had IMPM with focal carcinoma change (the neoplastic cyst size $2.5 \mathrm{~cm}$ ) had recurrence of the pancreatic remnant, 5 years after distal pancreatectomy and splenectomy. She underwent chemotherapy and radiotherapy and was well at last follow-up, 72 months after pancreatic resection.

\section{Discussion}

Examination of this small case series taught us useful information regarding cystic neoplasms of the pancreas. First, the prognosis of frank malignancy is poor even after an intentionally curative resection. Second, resection of premalignant or malignant cystic tumors may reduce the chance of tumor transformation into invasive cancer and enhance survival. Third, pancreatic resection may be performed safely by experienced surgical teams. The first author has performed more than 100 pancreaticoduodenectomies during his surgical career with $2 \%$ mortality and $44 \%$ morbidity rates [18]. Familiarity contributed greatly to rational decision making when unexpected findings were encountered. Traditionally, surgery is recommended in symptomatic cysts, large cysts, and suspected premalignant or malignant cystic neoplasms [6, 19-22]. However, when a decision has to be made for whether operation should be performed or not in high risk patients e.g. very old age or patients with serious comorbidity, the likelihood of malignancy and the surgeons' experience should be factors included into the decision. Pancreatic resection is a major operation, which may result in serious postoperative complications, even in centers with experienced surgeons and in high-volume surgical centers [13]. Of the 16 patients in our case series, 14 had definite indications for surgery by criteria of symptoms, size, and imaging appearance of the cystic neoplasms suggestive of premalignancy or malignancy. The remaining two patients had small cysts measuring 2.2 and $2.5 \mathrm{~cm}$ in diameter when first diagnosed with cystic neoplasms of the pancreas. The first patient, a woman aged 48 years, had a $2.2 \mathrm{~cm}$ cyst at the uncinate process of the pancreas with mild symptoms of epigastric discomfort. She was followed for 6 years until there were definite changes of the pancreatic lesion on her most recent CT with thickening of the cyst wall and demonstration of new intracystic nodules. Pylorus preserving pancreaticoduodenectomy was performed and the 
pathological reports revealed IPMN with moderate to severe dysplasia. She was well when this manuscript was prepared, 17 months after surgery. A second patient, a woman aged 69 years, had a $2.5 \mathrm{~cm}$ asymptomatic cyst on the body of her pancreas. Although the indication for surgery was not reached by size $(<3 \mathrm{~cm})$, surgery was performed without delay because of ERCP diagnosis of dilated pancreatic duct with intraductal filling defects [23]. She underwent distal pancreatectomy with splenectomy. The pathological reports were IPMN with focal carcinoma changes. She had recurrent carcinoma at the pancreatic remnant 5 years later and underwent chemotherapy and radiotherapy. She was alive and well at the time of preparing this manuscript, 72 months after pancreatic resection.

Regarding surgical techniques for pancreatic resection, we had acceptable results with low postoperative complications and no perioperative mortality. We had demonstrated in our previous reports of pancreaticoduodenectomy, that meticulous surgical techniques were important when performing these complex surgical procedures. Furthermore, external drainage of the pancreatic remnant, with an external pancreatic stent, may help lessen the occurrence of a postoperative pancreatic fistula [18, 24, 25]. When performing a distal pancreatectomy, the main pancreatic duct at the pancreatic stump must be identified and ligated to prevent a postoperative pancreatic fistula. The authors routinely used double suture-ligations of the main pancreatic duct with nonabsorbable suture number $4-0$. Of the 8 patients who underwent distal pancreatectomy, 6 also had splenectomy and 2 had not. The advantages of keeping the spleen intact were preserving its immunological functions and avoidance of creating a raw surface and potential space after splenectomy, which might be associated with postoperative fluid collection or infection [26]. However, splenic preserving distal pancreatectomy should be conducted only when technically feasible and the oncologic margins are not compromised.

Efforts to obtain a definite preoperative diagnosis of cystic neoplasms of the pancreas included imaging appearance by EUS, CT, and MRI; cystic fluid cytology and measurement of CEA, CA19-9 levels; and of measurement of serum biomarkers, e.g. CEA, CA19-9 [3, 8, 27]. However, definite preoperative diagnosis was still inconclusive in a large number of asymptomatic cysts incidentally found during abdominal imaging. The medical and surgical teams discuss the cases and come to a decision of whether to resect or to observe. This creates a continuing dilemma [28]. Current evidence-based reviews support safe observation for asymptomatic cysts $<2 \mathrm{~cm}$ with no demonstrable intracystic nodule on CT or MRI [27, 29]. Resection is recommended when changes or progression in size, symptoms, serum tumor markers, imaging appearance, and cystic fluid analysis are found during observation [27, 29, 30].

In conclusion, surgical treatment of cystic neoplasms of the pancreas has been presented. Definite indications for surgery were obtained in symptomatic cysts, large cysts, or strong imaging appearances suggesting malignancy $(\mathrm{n}=14)$. In two asymptomatic small cysts, resections were performed and revealed premalignant and malignant pathology. We concluded also that pancreatic resection should be performed for symptomatic and/or large cystic neoplasms. Imaging appearance suggestive of premalignancy or malignancy is a definite indication for surgery. A small asymptomatic cyst may be safely observed and followed-up. Any changes or progression of the cyst during the observation should result in resection. Meticulous surgical techniques are important during pancreatic resections for these cystic neoplasms in order to lessen postoperative complications.

\section{Acknowledgments}

The authors have not received any financial support from organizations or manufacturers of surgical instruments and have no conflicts of interest to declare.

\section{References}

1. Sohn TA, Yeo CJ, Cameron JL, Iacobuzio-Donahue CA, Hruban RH, Lillemoe KD. Intraductal papillary mucinous neoplasms of the pancreas : an increasingly recognized clinicopathologic entity. Ann Surg. 2001; 234:313-21.

2. Le Borgne J, de Calan L, Partensky C. Cystadenomas and cystadenocarcinomas of the pancreas: a multiinstitutional retrospective study of 398 cases. French Surgical Association. Ann Surg. 1999; 230: $152-61$.

3. Spence RA, Dasari B, Love M, Kelly B, Taylor M. Overview of the investigation and management of cystic neoplasms of the pancreas. Dig Surg. 2011; 28: 386-97.

4. Abe H, Kubota K, Mori M, Miki K, Minagawa M, 
Noie T, et al. Serous cystadenoma of the pancreas with invasive growth: benign or malignant? Am J Gastroenterol. 1998; 93:1963-6.

5. Strobel O, Z'Graggen K, Schmitz-Winnenthal FH, Friess H, Kappeler A, Zimmermann A, et al. Risk of malignancy in serous cystic neoplasms of the pancreas. Digestion. 2003; 68:24-33.

6. Fernandez-del Castillo C, Targarona J, Thayer SP, Rattner DW, Brugge WR, Warshaw AL. Incidental pancreatic cysts: clinicopathologic characteristics and comparison with symptomatic patients. Arch Surg. 2003; 138:427-33.

7. Keswani RN, Brentrem DJ, Hayman A, Zhu B, Lin X. Clinical characteristics and outcomes of surgically resected mucinous nonneoplastic cysts of the pancreas. Pancreas. 2012; 41:813-5.

8. Nadig SN, Pedrosa I, Goldsmith JD, Callery MP, Vollmer CM. Clinical implications of mucinous nonneoplastic cysts of the pancreas. Pancreas. 2012; 41:441-6.

9. Federle MP, McGrath KM. Cystic neoplasms of the pancreas. Gastroenterol Clin North Am. 2007; 36: 365-76.

10. Hodgkinson DJ, ReMine WH, Weiland LH. A clinicopathologic study of 21 cases of pancreatic cystadenocarcinoma. Ann Surg. 1978; 188:679-84.

11. Warshaw AL, Compton CC, Lewandrowski K, Cardenosa G, Mueller PR. Cystic tumors of the pancreas. New clinical, radiologic, and pathologic observations in 67 patients. Ann Surg. 1990; 212: 432-43.

12. Ridder GJ, Maschek H, Klempnauer J. Favourable prognosis of cystadeno-over adenocarcinoma of the pancreas after curative resection. Eur J Surg Oncol. 1996; 22:232-6.

13. Vin Y, Sima CS, Getrajdman GI, Brown KT, Covey A, Brennan MF, et al. Management and outcomes of postpancreatectomy fistula, leak, and abscess: results of 908 patients resected at a single institution between 2000 and 2005. J Am Coll Surg. 2008; 207: 490-8.

14. Carpizo DR, Allen PJ, Brennan MF. Current management of cystic neoplasms of the pancreas. Surgeon. 2008; 6:298-307.

15. Lee KS, Sekhar A, Rofsky NM, Pedrosa I. Prevalence of incidental pancreatic cysts in the adult population on MR imaging. Am J Gastroenteral. 2010; 105: 2079-84.

16. Gorin AD, Sackier JM. Incidental detection of cystic neoplasms of the pancreas. MD Med J. 1997; 46:79-82.
17. Bassi C, Dervenis C, Butturini G, Fingerhut A, Yeo C, Izbicki J, et al. Postoperative pancreatic fistula: an international study group (ISGPF) definition. Surgery. 2005; 138:8-13.

18. Sriussadaporn S, Sriussadaporn S, Pak-Art R, Kritayakirana K, Prichayudh S, Samorn P. Lessons learned from 100 personal consecutive cases of pancreaticoduodenectomy at a university hospital in Thailand. J Med Assoc Thai. 2013; 96:1147-58.

19. Brugge WR, Lauwers GY, Sahani D, Fernandez-del Castillo C, Warshaw AL. Cystic neoplasms of the pancreas. N Engl J Med. 2004; 351:1218-26.

20. Spinelli KS, Fromwiller TE, Daniel RA, Kiely JM, Nakeeb A, Komorowski RA, et al. Cystic pancreatic neoplasms: observe or operate. Ann Surg. 2004; 239: 651-7.

21. Goh BK, Tan YM, Cheow PC, Chung YF, Chow PK, Wong WK, et al. Cystic lesions of the pancreas: an appraisal of an aggressive resectional policy adopted at a single institution during 15 years. Am J Surg. 2006; 192:148-54

22. Moesinger RC, Talamini MA, Hruban RH, Cameron JL, Pitt HA. Large cystic pancreatic neoplasms: pathology, resectability, and outcome. Ann Surg Oncol. 1999; 6:682-90.

23. Tanaka M, Chari S, Adsay V, Fernandez-del Castillo C, Falconi M, Shimizu M, et al. International consensus guidelines for management of intraductal papillary mucinous neoplasms and mucinous cystic neoplasms of the pancreas. Pancreatology. 2006; 6:17- 32.

24. Sriussadaporn S, Prichayudh S, Sriussadaporn S, Kritayakirana K, Pak-art R. Pylorus preserving pancreaticoduodenectomy with low incidence of early delayed gastric emptying. J Med Assoc Thai. 2007; 90:82-8.

25. Sriussadaporn S, Pak-Art R, Sriussadaporn S, Kritayakirana K, Prichayudh S. Pancreaticoduodenectomy with external drainage of the pancreatic remnant. Asian J Surg. 2008; 31:167-73.

26. Warshaw AL. Distal pancreatectomy with preservation of the spleen. J Hepatobiliary Pancreat Sci. 2010; 17: 808-12.

27. Planner AC, Anderson EM, Slater A, Phillips-Hughes J, Bungay HK, Betts M. An evidence-based review for the management of cystic pancreatic lesions. Clin Radiol. 2007; 62:930-7.

28. Liao T, Velanovich V. Asymptomatic pancreatic cysts : a decision analysis approach to observation versus resection. Pancreas. 2007; 35:243-8.

29. Goh BK, Tan YM, Chung YF, Chow PK, Cheow PC, 
Thng $\mathrm{CH}$, et al. Pancreatic cysts: a proposed management algorithm based on current evidence. Am J Surg. 2007; 193:749-55.

30. Salvia R, Malleo G, Marchegiani G, Pennacchio S,
Paiella S, Paini M, et al. Pancreatic resections for cystic neoplasms: from the surgeon's presumption to the pathologist's reality. Surgery. 2012; 152 (3 Suppl 1):S135-42. 